\title{
¿Será sólo una cuestión de frío?
}

\author{
Maria João Teles, Ana Luisa Neves, Joana Almeida.
}

Unidade de Saúde Familiar Camélias. Vila Nova de Gaia (Portugal).

\section{Correspondencia:}

Maria João Teles.

Rua do Loureiro, $n^{\circ} 251$, Madalena. 4410-757 - Vila Nova de Gaia (Portugal).

Correo electrónico:

maryajoaoteles@gmail.com

Este trabajo ha sido presentado como comunicación oral en el $13^{\circ}$ Encontro de Medicina Geral e Familiar do Alto Minho (Arcos de Valdevez, del 31 de mayo al 2 de junio de 2012) y en forma de póster en el WONCA Europe Conference 2012 (Viena, del 4 al 7 de julio de 2012).

Recibido el 25 de octubre de 2013.

Aceptado para su publicación el 9 de enero de 2014

\section{RESUMEN}

El síndrome de CREST es una variante de la esclerodermia y se caracteriza por calcinosis, fenómeno de Raynaud, cambio de la motilidad esofágica, esclerodactilia y telangiectasias. Normalmente, la afectación cutánea se limita a las extremidades distales, la progresión es más lenta y el pronóstico más favorable que en la esclerodermia, pudiendo no existir compromiso visceral. El objetivo de este trabajo es relatar un caso clínico de síndrome de CREST, enfocando la importancia del médico de familia en la valoración de las señales y síntomas presentados por los pacientes, permitiendo un diagnóstico y una orientación terapéutica adecuados.

Palabras clave: Enfermedad de Raynaud. Síndrome CREST. Atención Primaria de Salud.

\section{ABSTRACT}

\section{Could it just be a matter of cold?}

The CREST syndrome is a variant of scleroderma characterized by calcinosis, Raynaud's phenomenon, esophageal dysfunction, sclerodactyly and telangiectasias. Skin affection is usually limited to distal extremities, it progresses more slowly and its prognosis is more favorable than in scleroderma, with the possibility of no visceral involvement. The aim of this paper is to present a clinical case of CREST syndrome, highlighting the relevance of the family physician in the assessment of the patient's signs and symptoms, thus allowing adequate diagnosis and therapeutic guidelines.

Keywords: Raynaud Disease. CREST Syndrome. Primary Health Care.

\section{INTRODUCCIÓN}

En los años 60, Carr et al. y Schimke et al. describieron el síndrome CREST como una variante de la esclerodermia sistémica, siendo más limitada, indolente y caracterizada por calcinosis, fenómeno de Raynaud, alteración de la motilidad esofágica, esclerodactilia y telangiectasias ${ }^{1}$. Normalmente, sólo tres de estas cinco características son necesarias para establecer el diagnóstico ${ }^{2}$. De una manera general, el sexo femenino es el más afectado ${ }^{1,3,4}$, en una proporción de 3:1. Las manifestaciones comienzan generalmente entre la tercera y la cuarta décadas de la vida ${ }^{3,4} \mathrm{y}$, con frecuencia, hay historia de fenómenos de Raynaud de varios años de evolución ${ }^{1,4}$. No hay pruebas diagnósticas específicas ${ }^{3}$, pero los anticuerpos anti-centrómero son característicos, y están presentes en más del $50 \%$ de los casos ${ }^{1}$. La velocidad de hemosedimentación puede estar ligeramente aumentada y el hemograma mostrar anemia de enfermedad crónica ${ }^{3}$. El pronóstico es habitualmente mejor que en la esclerosis sistémica ${ }^{1}$. El objetivo de este trabajo es relatar un caso clínico de síndrome CREST, enfocando la importancia de un diagnóstico y una orientación terapéutica adecuados. 


\section{OBSERVACIONES CLÍNICAS}

Paciente del sexo femenino, de 38 años de edad, sin antecedentes personales de interés, medicada con anticonceptivo oral, que había comenzado tres meses antes.

En mayo de 2008 acude a la consulta de su médico de familia refiriendo que, desde marzo de 2007, presentaba crisis de alteración de coloración de la piel del tercero y cuarto dedos de las manos, que se manifestaban con el frío. Primero los dedos se le ponían blancos durante 15-20 minutos y después se ponían azules y posteriormente rojos. Desde hacía años había notado inflamación en ambas manos sin rigidez matutina y pirosis tras la ingesta de alimentos.

Al examen se encuentra leve atrofia de la piel, sin otras alteraciones.

\author{
Enfermedades del tejido conectivo \\ Esclerosis sistémica variante difusa \\ Esclerosis sistémica variante localizada (CREST) \\ Enfermedad mixta del tejido conectivo \\ Lupus eritematoso sistémico \\ Síndrome de Sjögren \\ Dermatomiositis
}

Fármacos y agentes tóxicos

Bloqueantes beta

Ergotamínicos

Agentes quimioterápicos (bleomicina, carboplatino, vinblastina, etc.)

Ciclosporina

Interferón a y b

Cocaína

Nicotina

Policloruro de vinilo

Enfermedades endocrinas

Hipotiroidismo

Feocromocitoma

Síndrome carcinoide

Traumatismos

Síndrome del desfiladero torácico (unilateral)

Aneurisma cubital

Lesión por vibraciones

\title{
Anomalías en vasos sanguíneos
}

Tromboangeítis obliterante

Embolia periférica

Vasculitis (enfermedad de Takayasu, arteritis de células gigantes)

\section{Enfermedades hematológicas y tumores}

Crioglobulinemia

Paraproteinemias

Síndromes mielo/linfoproliferativos

Tabla 1. Causas de fenómeno de Raynaud secundario 
Con la sospecha diagnóstica de fenómeno de Raynaud, sin poder distinguir entre primario o secundario, se efectuó un estudio analítico que mostró anemia (hemoglobina de $11,9 \mathrm{mg} / \mathrm{dl}$ ), siendo el resto del hemograma, la eritrosedimentacón, la glicemia, la creatinina, las proteínas totales y fraccionadas, el tiempo de protrombina y las enzimas hepáticas normales. Presentó un título de anticuerpos anti-nucleares mayor de 1/80, moteado nuclear y anticentrómero positivos.

Se derivó a la consulta de Reumatología, donde se realizó la hipótesis diagnóstica de síndrome CREST. En esta etapa se realizó una capilaroscopia del lecho ungueal, que mostró una disminución de capilares en parches, dilataciones capilares y megacapilares. También se realizó una radiografia del tórax, que ha sido normal, y un esofagograma que mostró un esófago algo afinado, aunque sin otras alteraciónes. La biopsia de la piel confirmó el diagnóstico clínico de esclerodermia.

Fue medicada con nifedipina y pentoxifilina, que suspendió por intolerancia. Tras confirmarse el diagnóstico de síndrome CREST se instituyó tratamiento con metotrexate.

En enero de 2011 comenzó con episodios de artralgias en los miembros superiores que fueron interpretadas en este contexto. Actualmente, la paciente es seguida por su médico de familia con revisiones periódicas en la consulta de Reumatología.

\section{COMENTARIOS}

El síndrome CREST pertenece al grupo de las esclerodermias, que origina un engrosamiento y endurecimiento del tejido conjuntivo en varias localizaciones del cuerpo ${ }^{2}$. Puede ser extremadamente variable, y un paciente puede o no presentar todas las características en determinando momento ${ }^{5}$. El $70 \%$ de los enfermos refieren el fenómeno de Raynaud como queja inicial, ocurriendo la tríada palidez-cianosis-eritema, más frecuentemente en las manos y pies, con duración de pocos minutos hasta varias horas, como en el caso presentado. Ante un paciente con fenómeno de Raynaud diagnosticado clínicamente tenemos que distinguir entre primario, cuando no se identifica una causa subyacente; y secundario, si aparece en el contexto de otras enfermedades, reumáticas o de otra ín- dole, incluyendo enfermedad vascular extrínseca, alteraciones endocrinas y enfermedades hematológicas. Hay numerosas causas de fenómeno de Raynaud secundario (tabla 1). Debemos pensar en esta posibilidad ante un inicio posterior a los 30 años, episodios intensos, dolorosos, asimétricos o relacionados con lesiones isquémicas, precipitados por cambios posturales o cuando los pacientes presentan criterios de una enfermedad del tejido conectivo asociada.

La observación de la textura y localización de la piel endurecida puede también contribuir para el diagnóstico. Esta paciente tenía sólo leve atrofia de la piel. Generalmente, la rigidez cutánea comienza en los dedos de las manos. A nivel esofágico, la principal alteración es la existencia de atrofia y fibrosis de la musculatura esofágica, originando síntomas de reflujo ${ }^{3}$.

El abordaje del síndrome CREST debe ser dirigido a la sintomatología presentada. En este sentido, para controlar el fenómeno de Raynaud se propone la reducción del vasoespasmo, evitar el estrés o el frio y la prescripción de bloqueadores de los canales del calcio (por ejemplo, nifedipina o diltiazem). Los anti-inflamatorios no esteroideos se muestran eficaces en el control de las artralgias y las medidas anti-reflujo permiten controlar la dismotilidad esofágica ${ }^{3}$.

La atención y seguimiento del médico con respecto a las quejas y preocupaciones de sus pacientes es muy importante. La identificación y derivación precoces de determinados casos clínicos, son de primordial importancia para su control, contribuyendo al bienestar y calidad de vida de los enfermos.

\section{BIBLIOGRAFÍA}

1. Carneiro AC, Barbosa IP, Chaves FC. Síndrome de CREST e hipertensão pulmonar - prognóstico sombrio. Acta Med Port. 2004; 17 (5): 409-14.

2. Nakae R, Idei M, Kumano K, Okita S, Yamane K. Intracranial aneurysms in patients with CREST syndrome. Neurol Med Chir (Tokyo). 2009; 49 (9): 402-6.

3. Turtelli C, Silva H. Qual o seu diagnóstico? Radiol Bras 2002; 35 (6): V-VI.

4. Almeida MS, Carvalho LL, Arcoverde J, Capucho L, Monteiro C, Fontanelle G. Calcinose distrófica de vulva em paciente com Síndrome CREST. Acta Reumatol Port. 2010; 35: 264-6.

5. Raja MS, Marshall T, Burton BJ. Acute central retinal artery occlusion presenting as CREST syndrome: a case report. Cases J. 2009; 2 (1): 9 